\title{
Consentimiento informado en Medicina Práctica clínica e investigación biomédica
}

Rodrigo López, Patricio Vega.

División de Anestesiología - Centro de Bioética

Facultad de Medicina P. Universidad Católica de Chile

El consentimiento informado es, en la actualidad, un proceso ineludible tanto de la práctica médica como de la investigación biomédica. No sólo responde a necesidades éticas, para hacer a la persona partícipe en el proceso terapéutico, sino que respeta prescripciones legales.

Sin embargo, existen numerosas dificultades prácticas al momento de llevar a cabo un adecuado proceso de consentimiento informado. Las adecuadas habilidades de comunicación, así como un mejor entrenamiento en identificar información relevante y pacientes con alteraciones de la competencia, debiesen ser incorporadas en la formación de los médicos de manera más explícita; paralelamente, es necesaria más investigación respecto de los mejores métodos de entregar información a los pacientes y sujetos de investigación. 


\section{Informed consent for clnical practice and clinical research}

The authors present and discuss relevant issues regarding the process and the document of informed consent. Requirements by laws in Chile are included. Special emphasis is placed on the significance of the informed consent process for the education of patients, physicians and investigators. Some limitations in the administration of informed consent and possible solutions are presented. 


\section{Introducción}

Actualmente es indudable que la práctica médica ha cambiado de un paradigma paternalista, en que el médico dictaminaba las conductas a implementar en aras del bienestar del paciente, a un modelo más participativo en que tanto el médico como el paciente deciden juntos lo que resulta más aconsejable en cada caso particular. Es por esto que el proceso del consentimiento informado, que busca garantizar que la participación de un sujeto en alguna intervención médica o investigación sea voluntaria, ha cobrado cada vez más relevancia.

El consentimiento informado se sustenta en el reconocimiento fundamental que los adultos competentes tienen derecho a aceptar o rechazar intervenciones destinadas al cuidado de la salud, en base a los valores y objetivos de vida de la persona ${ }^{1}$.

Una de las principales fuentes de la deontología médica, el juramento hipocrático, no contiene mayores prescripciones referentes al consentimiento informado. En sus inicios el modelo de atención de la medicina era directivo, donde el médico dictaminaba lo mejor para el paciente, quien se limitaba a acatar y seguir las indicaciones. No es de extrañarse entonces que las primeras referencias al consentimiento informado, en la práctica clínica, no ocurrieran sino hasta el siglo XVIII en Inglaterra, cuando, producto de un mal resultado, un paciente entabló acciones civiles contra el médico. Éste, demostrando la existencia de un documento firmado en que el paciente reconoce los riesgos de la intervención propuesta, acepta la concreción de la misma $2,3,4$.

En investigación biomédica, una de las primeras veces en que se recurre a un documento de consentimiento informado fue en los experimentos de Walter Reed sobre la causa de la fiebre amarilla; para ser incluido entre los participantes era necesario firmar un contrato que explicaba los riesgos y establecía la voluntariedad de participar ${ }^{5,6}$. Sin embargo, no fue hasta las atrocidades cometidas en los campos de concentración de la Segunda Guerra Mundial que se hace patente la necesidad de contar con regulaciones éticas para la investigación biomédica, teniendo como uno de sus principales requerimientos la voluntariedad de participar, expresada en un documento de consentimiento informado. La primera de dichas normativas es el código de Nüremberg (1949), sus principios fueron actualizados y complementados en la Declaración de Helsinki (1964) y el Reporte Belmont (1978), y posteriormente adaptadas a condiciones propias de países en vías de desarrollo en las normas CIOMS (2002).

La práctica clínica y la investigación biomédica se carac- terizan actualmente por una mayor complejidad, sumado a pacientes que solicitan mayor información. En este contexto el proceso de consentimiento informado, entendido como la autorización voluntaria de un paciente o participante en investigación, que comprende los riesgos de los procedimientos diagnósticos, terapéuticos o científicos a los que se someterá, debiese considerarse como una forma de educar y respetar la autonomía del paciente. Los médicos debemos reconocer la importancia del proceso de consentimiento informado y capacitarnos para su correcta administración, lo que nos permitirá contar con pacientes informados y con expectativas realistas de los riesgos y beneficios de los procedimientos a los que se someterán $^{2}$.

\section{Algunas distinciones}

Es necesario evitar la tradicional confusión entre el proceso de consentimiento informado propiamente tal, del documento de consentimiento informado. Mientras el consentimiento informado es un proceso por el cual una persona debidamente informada acepta, o rechaza, su participación en alguna acción de salud previamente definida, el documento de consentimiento informado es sólo el registro de la ocurrencia de dicho proceso y constituye un medio de prueba de la decisión tomada ${ }^{7}$.

No todo proceso de consentimiento informado debe verse plasmado en un documento de consentimiento informado, ya que intervenciones de bajo riesgo, como la toma de sangre venosa, no necesitan la firma de ningún formulario. La ley chilena señala que debe existir un documento de consentimiento informado escrito, incluido en la ficha clínica del paciente señalando la información entregada y la aceptación o rechazo por el paciente, en el caso de: intervenciones quirúrgicas, procedimientos diagnósticos y terapéuticos invasivos y, en general, para la aplicación de procedimientos que conlleven un riesgo relevante y conocido para la salud del afectado ${ }^{8}$.

El consentimiento informado es un proceso mediante el cual la persona que va a someterse a una acción de salud decide participar voluntariamente. A través del consentimiento las personas ejercen su derecho a autodeterminación, salvaguardando el principio clásico de la autonomía de las personas, y por ello es exigido en numerosas legislaciones, incluida la chilena. Algunos autores afirman que el consentimiento es la mejor manera de demostrar respeto por las personas que van a ser sometidas a acciones de salud ${ }^{9}, 10$.

En investigación el proceso de consentimiento comienza en el contacto inicial con el participante del proyecto 
mediante la entrega de información de manera comprensible y no sesgada de la investigación que se realizará, respondiendo preguntas a medida que surjan durante la investigación (reforzando el concepto de proceso que acompaña al participante a lo largo de la investigación) y asegurando que cada persona comprende adecuadamente lo que va a suceder dentro de la investigación. De esta manera se evidencia el respeto por la dignidad y se reconoce la autonomía de las personas; por lo que es importante dar tiempo suficiente, incluyendo tiempo para consultar a quienes el participante considere importante (familia, amigos, etc.) para tomar una decisión, libre y sin coerción 7 .

El consentimiento debe ser informado, voluntario y realizado por una persona competente. Para considerar que una decisión fue tomada de manera autónoma es necesario cumplir con algunos requisitos. Primero, la persona que toma la decisión debe estar plenamente informada de la naturaleza (terapia o investigación) y consecuencia de las distintas alternativas; segundo, debe tomar la decisión de manera voluntaria, existiendo distintas visiones de lo que significa voluntariedad desde la mera falta de coacción externa a la coherencia con los valores de la persona; por último, la persona debe tener la capacidad de tomar decisiones, es decir, ser competente ${ }^{11,12}$.

Desde el punto de vista legal, son incompetentes los menores de edad, los interdictos por demencia y los sordomudos analfabetos. Dentro de las capacidades más utilizadas para evaluar la competencia destacan: comprender la información proporcionada, apreciar la situación y sus posibles consecuencias, razonar las alternativas y сотиnicar la decisión tomada ${ }^{13,14}$.

Estos criterios, si bien ampliamente utilizados en la práctica, han sido criticados por proporcionar demasiada relevancia a funciones cognitivas, desconociendo la importancia que tienen las emociones en el proceso de toma de decisiones. Alguien puede ser competente para decidir si se toma un medicamento o no, pero no serlo para decidir si participar o no en una investigación científica, porque se requieren distintos niveles de comprensión para una $u$ otra decisión; del mismo modo, las capacidades cognitivas pueden variar en el tiempo, como es el caso de un síndrome confusional agudo, por lo que la competencia de estos pacientes también puede fluctuar.

Los menores de edad, son incompetentes desde el punto de vista legal, y en los consentimientos que los atañen su participación debe ser subrogada por algún representante legal, usualmente los padres del menor. Sin embargo, desde el punto de vista ético se considera que ellos pue- den tener las capacidades necesarias para participar en el proceso de toma de decisiones, aún de modo parcial, por lo que no se debe asumir una incapacidad absoluta. De este modo, se contempla considerar la opinión de niños y adolescentes menores de 18 años por medio del proceso de asentimiento informado, el que debe adecuarse al nivel de desarrollo del menor. Esta misma aproximación debiera contemplarse para mayores de edad con capacidad de consentir disminuida; si bien pueden carecer competencia necesaria para consentir, muchas veces poseen las capacidades para participar en el proceso de toma de decisiones por medio del asentimiento.

Existen algunas excepciones a la obligatoriedad de efectuar el proceso de consentimiento informado antes de cualquier intervención en salud. Entre las más conocidas destacan la imposibilidad de obtener consentimiento (paciente sin la capacidad de consentir y no se encuentra disponible ningún representante), siempre y cuando la atención constituya una emergencia médica que no pueda ser retrasada y que dicha intervención busque procurar directamente el bienestar del paciente y se adopten las medidas apropiadas para garantizar la protección de la vida; en cualquier caso, la intervención puede ser consentida a posteriori una vez que el paciente recupere las facultades mentales o se localice a algún representante válido ${ }^{8}$.

Otra excepción al consentimiento informado ocurre cuando la intervención de salud no busca el bienestar del individuo, sino que está orientada al bien común, como son acciones de salud pública. No es necesario que el paciente consienta para proceder con el reporte de una enfermedad de notificación obligatoria. En este sentido las vacunas constituyen un caso interesante porque, si bien su aplicación constituye un interés de salud pública, la negativa de los sujetos o sus representantes ha sido esgrimida como razón para la no administración de manera cada vez más frecuente. Este tema sigue siendo altamente debatido en la bioética actual, sin haberse alcanzado aún consenso ${ }^{15}$.

En el caso de investigaciones biomédicas que no impliquen riesgos para los participantes y en las que no se desea recabar información sobre la identificación de los mismos, como sería el caso de encuestas con una muestra no intencionada o el estudio retrospectivo de bases de datos que no incluyan la identidad de los sujetos, se podría solicitar una dispensa de consentimiento informado. No obstante, se debe confirmar que la revelación de la información para los participantes no posee ninguna implicancia posterior. 


\section{Disposiciones legales}

Existen leyes y reglamentos que establecen requisitos del proceso de consentimiento informado. En estos requisitos subyace el valor de respeto por la autonomía de la persona, lo cual es profundamente apreciado en la sociedad actual. La autonomía en este contexto se entiende como el derecho del paciente de definir sus propias metas $\mathrm{y}$ tomar decisiones que permitan alcanzarlas ${ }^{16}$.

Son numerosas las disposiciones legales actualmente vigentes en Chile que regulan el proceso de consentimiento informado, tanto para la práctica clínica como para la investigación en salud.

La ley 20.548 que "Regula los derechos y deberes que tienen las personas en relación con acciones vinculadas a su atención de salud", publicada en 2012, en su artículo 10 señala:

"Toda persona tiene derecho a ser informada, en forma oportuna y comprensible, por parte del médico u otro profesional tratante, acerca del estado de su salud, del posible diagnóstico de su enfermedad, de las alternativas de tratamiento disponibles para su recuperación y de los riesgos que ello pueda representar, así como del pronóstico esperado, y del proceso previsible del postoperatorio cuando procediere, de acuerdo con su edad y condición personal y emocional.

Cuando la condición de la persona, a juicio de su médico tratante, no le permita recibir la información directamente o padezca de dificultades de entendimiento o se encuentre con alteración de conciencia, la información a que se refiere el inciso anterior será dada a su representante legal, o en su defecto, a la persona bajo cuyo cuidado se encuentre. Sin perjuicio de lo anterior, una vez que haya recuperado la conciencia y la capacidad de comprender, deberá ser informada en los términos indicados en el inciso precedente.

Tratándose de atenciones médicas de emergencia o urgencia, es decir, de aquellas en que la falta de intervención inmediata e impostergable implique un riesgo vital o secuela funcional grave para la persona y ella no esté en condiciones de recibir y comprender la información, ésta será proporcionada a su representante o a la persona a cuyo cuidado se encuentre, velando porque se limite a la situación descrita. Sin perjuicio de lo anterior, la persona deberá ser informada, de acuerdo con lo indicado en los incisos precedentes, cuando a juicio del médico tratante las condiciones en que se encuentre lo permitan, siempre que ello no ponga en riesgo su vida. La imposibilidad de entregar la información no podrá, en ningún caso, dilatar o posponer la atención de salud de emergencia o urgencia.
Los prestadores deberán adoptar las medidas necesarias para asegurar la adecuada confidencialidad durante la entrega de esta información, así como la existencia de lugares apropiados para ello". 8

Se establece así la obligatoriedad de entregar la información pertinente a los pacientes. A continuación, se establece la necesidad de contar con el consentimiento antes de ejecutar cualquier intervención en salud, como está estipulado en el artículo 14 de la misma ley:

"Toda persona tiene derecho a otorgar o denegar su voluntad para someterse a cualquier procedimiento o tratamiento vinculado a su atención de salud, con las limitaciones establecidas en el artículo 16.

Este derecho debe ser ejercido en forma libre, voluntaria, expresa e informada, para lo cual será necesario que el profesional tratante entregue información adecuada, suficiente y comprensible, según lo establecido en el artículo 10 .

En ningún caso el rechazo a tratamientos podrá tener como objetivo la aceleración artificial de la muerte, la realización de prácticas eutanásicas o el auxilio al suicidio.

Por regla general, este proceso se efectuará en forma verbal, pero deberá constar por escrito en el caso de intervenciones quirúrgicas, procedimientos diagnósticos y terapéuticos invasivos y, en general, para la aplicación de procedimientos que conlleven un riesgo relevante y conocido para la salud del afectado. En estos casos, tanto la información misma, como el hecho de su entrega, la aceptación o el rechazo deberán constar por escrito en la ficha clínica del paciente y referirse, al menos, a los contenidos indicados en el inciso primero del artículo 10. Se presume que la persona ha recibido la información pertinente para la manifestación de su consentimiento, cuando hay constancia de su firma en el documento explicativo del procedimiento o tratamiento al cual deba someterse." 8

La investigación científica que involucra personas como sujetos de investigación está regulada por la ley 20.120 "Sobre la investigación científica en el ser humano, su genoma, y prohíbe la clonación humana", publicada en septiembre de 2006. En el artículo 11 estable que:

“Toda investigación científica en un ser humano deberá contar con su consentimiento previo, expreso, libre e informado, o, en su defecto, el de aquel que deba suplir su voluntad en conformidad con la ley.

Para los efectos de esta ley, existe consentimiento informado cuando la persona que debe prestarlo conoce los aspectos esenciales de la investigación, en especial su finalidad, beneficios, riesgos y los procedimientos o 
tratamientos alternativos. Para ello deberá habérsele proporcionado información adecuada, suficiente y comprensible sobre ella. Asimismo, deberá hacerse especial mención del derecho que tiene de no autorizar la investigación o de revocar su consentimiento en cualquier momento y por cualquier medio, sin que ello importe responsabilidad, sanción o pérdida de beneficio alguno.

El consentimiento deberá constar en un acta firmada por la persona que ha de consentir en la investigación, por el director responsable de ella y por el director del centro o establecimiento donde ella se llevará a cabo, quien, además, actuará como ministro de fe.

En todo caso, el consentimiento deberá ser nuevamente solicitado cada vez que los términos o condiciones en que se desarrolle la investigación sufran modificaciones, salvo que éstas sean consideradas menores por el Comité Ético Científico que haya aprobado el proyecto de investigación." 17

Ahora bien, es necesario recordar que en la práctica médica también pueden aplicar normativas como la ley 19.628, "sobre la protección de la vida privada o protección de datos de carácter personal". ${ }^{18}$

Para el caso de la investigación científica, la ley 20.850, "Ley Ricarte Soto", establece requerimientos referentes al consentimiento informado. En dicha ley se señala que en investigaciones biomédicas que utilicen productos farmacéuticos o dispositivos de uso médico (ej.: prótesis) que no cuenten con registro sanitario o bien, contando con éste se pretenda un uso distinto al registrado, necesitan una autorización especial para uso provisional con fines de investigación, Para solicitar tal autorización en el instituto de salud pública se requiere el protocolo de investigación, el formato de consentimiento informado y una póliza de seguros entre otros ${ }^{19}$.

La Asociación Médica Mundial (WMA enfatiza que los pacientes tienen derecho a información y a la autodeterminación. Esta asociación en su declaración de Helsinki, al igual que otros códigos internacionales de ética de investigación, extiende este derecho al ámbito de la investigación biomédica, señalando la importancia del consentimiento informado en la investigación a nivel global ${ }^{20,21}$. La Asociación Médica Americana, considera al consentimiento informado como una política básica que constituye una obligación ética y legal que los médicos deben cumplir $^{22}$.

\section{¿Qué información se debe entregar en el proceso de consentimiento informado?}

Debido a la diferencia en los objetivos entre la práctica clínica y la investigación biomédica, la información que se debe proporcionar al paciente que va a ser sometido a una intervención en salud, difiere de manera significativa de la que se debe entregar a un sujeto participante en un proyecto de investigación. Mientras que en el primer caso la intervención está destinada al bienestar del paciente, en el segundo caso el estudio busca aumentar el conocimiento que la sociedad tiene sobre un determinado fenómeno. Es por esto que los consentimientos de investigación, en general, proporcionan mayor información.

Sin embargo, no existe consenso entre médicos e investigadores en relación a la extensión, nivel de detalles y metodología que debe emplearse al entregar información durante el proceso de consentimiento. ${ }^{23,24,25}$

Lo más aceptado es que durante el proceso de consentimiento informado en la práctica clínica, el paciente debiese recibir una descripción del procedimiento en cuestión (de qué se trata), así como de la duración del mismo. Se debe establecer claramente cuáles son los objetivos de dicha intervención, sea de diagnóstico o tratamiento, así como los riesgos potenciales y los beneficios esperados. Por último, el paciente debe conocer las alternativas de tratamiento (incluyendo los riesgos y beneficios de dichas alternativas), así como los procedimientos necesarios en caso que desistir de la intervención ${ }^{26}$. Actualmente aún no existe consenso sobre la obligatoriedad de informar sobre los costos derivados de la intervención. Mientras en un sistema de salud socializado, donde los costos son absorbidos por el estado sin afectar al paciente, esto parece ser innecesario, en sistemas de salud en que los costos derivan directa o indirectamente del paciente la información sobre los aspectos económicos parece ser imprescindible. Sin embargo, muchos médicos se desentiendan de esta parte, delegando la responsabilidad de averiguar por el costo económico al mismo paciente o su familia. Para el caso de proyectos de investigación se debe entregar más información porque la intervención no está orientada primariamente al bienestar del sujeto participante. Así, lo primero que debe ser comunicado es el propósito de la intervención, es decir, que se busca contestar una pregunta de investigación y que no constituye en ningún caso un tratamiento médico. Luego se debe informar sobre los objetivos del estudio en cuestión y describir los procedimientos a los que los sujetos serán sometidos, así como la duración de los mismos. Se deben explicitar los riesgos conocidos, dejando la posibilidad de efectos adversos no conocidos cuando la experiencia previa sea limitada, así como los potenciales beneficios que el participante podría obtener. En estudios 
en que la intervención se compare con algún placebo, se debe explicar que el sujeto puede ser asignado al grupo placebo, sin recibir el potencial beneficio esperado. Se debe informar de las posibles alternativas al estudio $y$, en caso de existir costos para el sujeto, estos deben explicitarse. Del mismo modo, se debe informar de eventuales compensaciones con que el investigador retribuya el tiempo y/o las molestias que causaron las intervenciones, así como de posibles seguros en caso de existir daños. Durante la ejecución de algunos estudios es posible conocer información relevante del sujeto participante y no resultaría adecuado no proporcionársela; en esos casos, el modo de contactar al investigador para conocer esa información debe estar claramente especificado. En casos en que resulte obligatorio para el investigador proporcionar la información (ej.: enfermedades infectocontagiosas o de alto riesgo), se debe advertir del eventual contacto por parte del investigador para informar dicha condición. Se debe comentar el modo en que se resguardará la confidencialidad de los participantes y, de ser imposible asegurar la no identificación de los datos, se debe pedir la autorización para revelar la identidad del sujeto. Por último, se deben proporcionar datos de contacto del investigador (incluyendo afiliación a universidad o centro de salud), en caso que el participante tenga dudas sobre el proyecto en sí, y del presidente del comité ético científico que aprobó la investigación, para preguntas sobre los derechos como sujeto de investigación ${ }^{26}$. El formulario de consentimiento finaliza con la declaración de participación voluntaria del sujeto, quien debe firmar dicho documento junto al investigador y al director de la institución en que ocurre la investigación, o su delegado, quien actúa como ministro de fe.

\section{Problemas prácticos al consentimiento informado}

Son numerosos los problemas que se han identificado para llevar correctamente a cabo un proceso de consentimiento informado. Entre los más nombrados se cuentan las situaciones de emergencia. Sin embargo, es necesario recordar que cuando el paciente no es capaz de consentir es posible eximirse de la obligación de completar todo el proceso. Ahora bien, existen casos en que el paciente tiene conservadas sus funciones cognitivas, pero la condición médica a la que se enfrenta hace del consentimiento informado una carga adicional. (Un paciente habla sobre la participación en la toma de decisiones a su ingreso al hospital: ... Ellos trataban de decirme lo que estaba pasando... Ésa era toda la participación que podía manejar en ese momento" ${ }^{27}$ ). En estos casos se debe requerir mayor participación a la familia u otro representante. En este mismo sentido, en algunas culturas no es propio de algunas personas participar en los procesos de toma de decisión, costumbre que está tan arraigada que estos mismos individuos prefieren abstenerse de dar su opinión. Preguntar por el parecer de estas personas podría suponer la paradoja que se vulnere su autonomía, justamente intentando salvaguardarla.

Otro de los problemas esgrimidos es la falta de tiempo, ya que con frecuencia el proceso tiene una duración más prolongada que lo disponible en la práctica clínica. Por su parte, los pacientes frecuentemente no comprenden los riesgos y alternativas de los procedimientos médicos y quirúrgicos ${ }^{28}$, y sus decisiones muchas veces se basan en la confianza que tienen en el médico más que en la información que se les entrega.

Es por esto que muchas instituciones tienen formularios en que se especifica toda la información requerida de manera escrita, pero estos no siempre incluyen información suficiente, lo que, sumado a la mala comprensión lectora de la población, hacen de esta alternativa una solución parcial $^{29,30}$.

Dificultades en la comprensión de la información también han sido descritas como problemas al proceso del consentimiento informado. Factores de riesgo para limitar la comprensión serían la mayor edad y el bajo nivel educacional ${ }^{16,26}$.

Las patologías neuropsiquiátricas también podrían ser un problema al momento de consentir. Sin embargo, establecer que esta población es incompetente sin una mayor evaluación podría constituir una discriminación injustificada $^{31}$.

El consentimiento para investigación implica el uso de conceptos a los que la población general está poco expuesta, como aleatorización, estudio ciego o placebo. Por otra parte, aunque la mayoría de los consentimientos informados de proyectos de investigación comienzan por reconocer la naturaleza experimental de la intervención, más del 50\% de las veces el sujeto de investigación cree que forma parte de un tratamiento médico. Esta confusión, denominada "error terapéutico", se asocia a una sobrevaloración de los posibles beneficios e infravaloración de los eventuales riesgos ${ }^{32}$.

En la actualidad existe una constante incorporación de nuevas tecnologías en la práctica médica. En respuesta a esto los programas de formación americanos de la ACGME (Accreditation Council for Graduate Medical Education) reconocen la necesidad de incluir competencias que van más allá de los conocimientos médicos y habilidades 
clínicas como, por ejemplo, el desarrollo de habilidades de comunicación ${ }^{33}$.

Las nuevas tecnologías representan desafíos para la realización del proceso de consentimiento informado y la manera en que esta información debe ser entregada a los pacientes. Por lo demás, las nuevas tecnologías se acompañan de riesgos difíciles de cuantificar, lo que dificulta la entrega de información en relación a riesgos, beneficios y posibles resultados. En este escenario, debemos considerar al consentimiento como un proceso que acompaña al paciente/participante a lo largo de la terapia o investigación, entregando constantemente nueva información que sea relevante para el paciente, haciendo de este proceso una instancia que permite educarlo y que ayuda a la toma de decisiones en salud ${ }^{1}$.

La presencia de diferencias culturales, personales y sociales plantean desafíos en el proceso de consentimiento. El respeto por la persona implica respetar los valores de las diferentes culturas y grupos, para lo cual en ciertos casos se deberá adaptar la información que se entregará. Valores como la lealtad, compasión y solidaridad pudiesen ser más importantes que la autonomía para ciertas personas y culturas. Además, en la toma de decisiones pudiese ser necesario incluir a familiares y miembros de la comunidad 34,35 .

El aumento del número de personas mayores de 65 años es un nuevo desafío al proceso de consentimiento. Este grupo etario con frecuencia usa los servicios de salud, tienen una alta prevalencia de enfermedades crónicas y puede presentar problemas cognitivos y físicos que dificulten el consentir. Médicos no entrenados en determinar la competencia de los pacientes para consentir, frecuentemente sólo reconocen la incapacidad en los casos en que el paciente rechaza un tratamiento médicamente recomendado o en casos de decisiones que impliquen riesgos. Los médicos e investigadores debiesen ser capaces de determinar la competencia de los pacientes entrenándose en el uso de herramientas validadas y útiles para este propósito o, en su defecto, solicitar ayuda a equipos capacitados en la determinación de competencias ${ }^{36}$.

\section{Posibles soluciones}

Se necesitan estrategias innovadoras para entregar a nuestros pacientes una medicina más segura, eficiente, basada en evidencia y que respete las preferencias individuales del paciente. En este contexto, el proceso de consentimiento informado debiese ser visto como una forma de educar a los pacientes en relación a los riesgos, beneficios y alternativas de los tratamientos e investigaciones propuestas y como una forma de promover la toma de decisiones en salud ${ }^{37}$.

Se ha demostrado que cuando los médicos son entrenados en estrategias de comunicación, los pacientes comprenden mejor los riesgos. Dedicar tiempo para explicar, usar estrategias prácticas que sinteticen y simplifiquen la información, contar con el apoyo de herramientas tecnológicas son formas que ayudan a alcanzar las metas éticas del proceso de consentimiento informado, favorecen la toma de decisiones compartidas, mejoran la comprensión y satisfacción de los pacientes $38,39,40,41,42$.

Para hacer frente a los múltiples problemas y desafíos del proceso de consentimiento se han planteado variadas soluciones. Una estrategia posible es una rigurosa discriminación de la información que el paciente necesita conocer para poder consentir libremente. Si bien es cierto que un mayor conocimiento podría derivar en una mejor apreciación de la situación, también es cierto que puede ser fuente de mayor confusión. Además de asegurarse que la información a entregar sea la adecuada, se debe procurar que el lenguaje sea comprensible considerando que no todas las personas comprenden el lenguaje médico. Contar con personas sin entrenamiento en cuidados de salud para "ajustar" la cantidad de información y el modo en que ésta es transmitida puede ser una buena idea, más aún cuando se trata de proyectos de investigación con diseños complejos ${ }^{30}$.

Un ejemplo de esto es un estudio en que se buscaba mejorar el proceso de consentimiento informado en intervenciones coronarias percutáneas. Se desarrolló un nuevo documento de consentimiento más simple, que incluía imágenes del procedimiento e incorporaba estimaciones individualizadas para el paciente de los resultados de la intervención y de los riesgos de complicaciones. Se estudió a 1.117 pacientes en 9 centros, 527 de ellos recibieron el nuevo consentimiento, y en este grupo se demostró una significativa mejor comprensión de propósito y riesgo del procedimiento, y mayor participación en la toma de decisiones. Además, estos pacientes discutieron más las opciones de tratamiento - en este caso stents - con sus médicos (58\% vs $31 \%$ ) en comparación con el grupo que recibió el documento de consentimiento estándar ${ }^{43}$.

Otra estrategia para entregar información es el uso de medios audiovisuales (videos, animaciones). Existe evidencia que este tipo de técnicas aumentan la retención de la información, que resulta necesaria para una apreciación cuidadosa de la situación y posibles consecuencias de las distintas alternativas propuestas. Corroborar el 
grado de comprensión de la información alcanzada por la persona puede ser una buena medida de seguridad. Así, la tradicional frase "dígame usted lo que entendió con sus propias palabras" puede resultar de gran utilidad en el proceso de consentimiento informado.

\section{Conclusión}

El consentimiento informado es, en la actualidad, un proceso ineludible tanto de la práctica médica como de la investigación biomédica. No sólo responde a necesidades éticas, para hacer a la persona partícipe en el proceso te- rapéutico, sino que respeta prescripciones legales.

Sin embargo, existen numerosas dificultades prácticas al momento de llevar a cabo un adecuado proceso de consentimiento informado. Las adecuadas habilidades de comunicación, así como un mejor entrenamiento en identificar información relevante y pacientes con alteraciones de la competencia, debiesen ser incorporadas en la formación de los médicos de manera más explícita; paralelamente, es necesaria más investigación respecto de los mejores métodos de entregar información a los pacientes y sujetos de investigación.

\section{Referencias}

1. GRADY C. Enduring and emerging challenges of informed consent. N Engl J Med. 2015; 372:855-862.

2. LECLERCQ WKG, KEULERS BJ, SCHELTINGA MRM, SPAUWEN PHM, VAN DER WILT G-J. A Review of Surgical Informed Consent: Past, Present, and Future. A Quest to Help Patients Make Better Decisions. World Journal of Surgery. 2010;34:1406-1415.

3. SLATER VS. BAKER AND STAPLETON. 95 Eng Rep. 860 (KB 1767).

4. CURRAN WJ, HALL MA, KAYE DH. Health care law, forensic science, and public policy, 4th ed. Little, Brown and Company. Boston, EEUU; 1990.

5. CUTTER L. Walter Reed, Yellow Fever, and Informed Consent. Mil Med. 2016;181:90-91.

6. MEHRA A. Politics of Participation: Walter Reed's Yellow-Fever Experiments. Virtual Mentor. 2009;11:326-330.

7. MONDRAGON-BARRIOS L. Consentimiento informado: una praxis dialógica para la investigación. Revista de Investigación Clínica; Órgano Del Hospital de Enfermedades de La Nutricion.2009;61,73-82.

8. Ley que "Regula los derechos y deberes que tienen las personas en relación con acciones vinculadas a su atención en salud". Ley $N^{\circ}$ 20.584. Diario Oficial de la República de Chile, Santiago, Chile, 24 de Abril de 2014. Disponible en <http://bcn. $\mathrm{cl} / 1 \mathrm{uw} 7 \mathrm{l}>$.
9. CHILDRESS J, BEAUCHAMP T. Principles of biomedical ethics. Oxford University Press, EEUU; 2013.

10. LEVINE R. Consent issues in human research. Encyclopedia of bioethics, ed. Warren T. Reich. New York, EEUU. 1995; 12411250.

11. MEISEL A, ROTH L. What we do and do not know about informed consent. JAMA. 1981;246:2473-2477.

12. LORDA S, CONCHEIRO P. El consentimiento informado: teoría y práctica. Med Clin (Barc). 1993;100:659-663.

13. GRISSO T, APPELBAUM P. Assessing competence to consent to treatment: a guide for physicians and other health professionals. Oxford University Press, New York, EEUU; 1998.

14. APPELBAUM P. Clinical practice. Assessment of patients' competence to consent to treatment. N Engl J Med. 2007;357:1834-1840.

15. ZIMMERMAN RK. Ethical analysis of HPV vaccine policy options. Vaccine. 2006;24:4812-4820.

16. FEDEN R, BEAUCHAMP T. A history and theory of informed consent. Oxford University Press, New York, EEUU; 1986.

17. Ley "Sobre la investigación científica en el ser humano, su genoma, y prohíbe la clonación humana" Ley $N^{\circ} 20.120$. Diario Oficial de la República de Chile, Santiago, Chile, 22 de Septiembre de 2006. Disponible en <http://bcn.cl/1uxxf>. 
18. Ley "Sobre protección de la vida privada" Ley N ${ }^{\circ} 19.628$ Diario Oficial de la República de Chile, Santiago, Chile, 28 de Agosto de 1999. Disponible en <http://bcn.cl/1uv2v>.

19. Ley que "Crea un sistema de protección financiera para diagnósticos y tratamientos de alto costo y rinde homenaje póstumo a don Luis Ricarte Soto Gallegos" Ley N ${ }^{\circ} 20.850$ Diario Oficial de la República de Chile, Santiago, Chile, 06 de Junio de 2015. Disponible en $<\mathrm{http}$ ://bcn.cl/1v7lo $>$.

20. WORLD MEDICAL ASSOCIATION. WMA Declaration of Lisbon on the rights of the patient. October 2005.

21. WORLD MEDICAL ASSOCIATION. WMA Declaration of Helsinki Ethical principles for medical research involving human subjects. October 2013.

22. The AMA Code of Medical Ethics' Opinions on Informing Patients. American Medical Association Journal of Ethics. 2012;14: 555-556.

23. MC MANUS PL, WHEATLEY KE. Consent and complications: risk disclosure varies widely between individual surgeons. Ann R Coll Surg Engl. 2003;85:79-82.

24. BOTTRELL MM, ALPERT H, FISCHBACH RL, EMANUEL LL. Hospital informed consent for procedure forms: facilitating quality patient-physician interaction. Arch Surg. 2000;135:26-33.

25. MCKNEALLY MF, IGNAGNI E, MARTIN DK, D’CRUZ J. The leap to trust: perspective of cholecystectomy patients on informed decision making and consent. J Am Coll Surg. 2004;199:51-57.

26. EMANUEL E, WENDLER D, GRADY C. What makes clinical research ethical? JAMA. 2000;283:2701-2711.

27. HÖGLUND AT, WINBALD U, ARNETZ B, ARNETZ JE. Scand J Caring Sci. 2010,24:482-489

28. FALAGAS ME, KORBILA IP, GIANNOPOULOU KP, KONDILIS BK, PEPPAS G. Informed consent: how much and what do patients understand? Am J Surg. 2009;198:420-435.

29. HOPPER KD, TENHAVE TR, TULLY DA, et al. The readability of currently used surgical/procedure consent forms in the United States. Surgery. 1998;123:496-503.

30. JEFFORD M, MOORE R. Improvement of informed consent and the quality of consent documents. Lancet Oncol. 2008;9:485-493.

31. PINALS D, APPELBAUM P. Ethical aspects of neuropsychiatric research with human subjects. Neuropsychopharmacology:
The Fifth Generation of Progress, ed. Kenneth L. Davis, Dennis Charney, Joseph T. Coyle, and Charles Nemeroff. American College of Neuropsychopharmacology, EEUU; 2002.

32. MILLER FG, JOFFE S. Evaluating the therapeutic misconception. Kennedy Inst Ethics J. 2006;16:353-366.

33. SEALS AA. Fellowship Training in Cardiology: Finding Synergies Between Academic Program Clinical Competencies and ACC-Developed Nonclinical Competencies. J Am Coll Cardiol. 2016;68:2376-2378.

34. TURNER L. From the local to the global: bioethics and the concept of culture. J Med Philos. 2005;30:305-320.

35. GOSTIN LO. Informed Consent, cultural sensitivity, and respect for persons. JAMA. 1995;274:844-845.

36. SESSUMS L, ZEMBRZUSKA H, JACKSON J. Does this patienthave medical decision-making capacity? JAMA. 2011;306:420-427.

37. President's commission for the study of ethical problems in medicine and biomedical and behavioral research. Making health care decisions: a report on the ethical and legal implications of informed consent in the patient-practitioner relationship. Washington DC: Library of Congress, EEUU. 1982.

38. KINNERSLEY P, PHILLIPS K, SAVAGE K, et al. Interventions to promote informed consent for patients undergoing surgical and other invasive healthcare procedures. Cochrane Database Syst Rev 2013;7:CD009445.

39. STACEY D, LÉGARÉ F, COL NF, et al. Decision aids for people facing health treatment or screening decisions. Cochrane Database Syst Rev 2014; 1:CD001431.

40. SCHENKER Y, MEISEL A. Informed consent in clinical care: practical considerations in the effort to achieve ethical goals. JAMA. 2011;305:1130-1131.

41. WOOLF SH, CHAN EC, HARRIS R, et al. Promoting informed choice: transforming health care to dispense knowledge for decision making. Ann Intern Med. 2005;143:293-300.

42. KRUMHOLZ HM. Informed consent to promote patient-centered care. JAMA. 2010;303:1190-1191.

43. SPERTUS S J, BACH R, BETHEA C, CHHATRIWALLA A, CURTIS J, GIALDE E, GUERRERO M, GOSCH K, JONES P, KUGELMASS A, LEONARD B, MCNULTY E, SHELTON $\mathrm{M}$, TING H, DECKER C. Improving the process of informed consent for percutaneous coronary intervention: patient outcomes from the Patient Risk Information Services Manager(ePRISM) study. Am Heart J. 2015;169: 